\title{
Focused Diagnostic Approach to A Patient with Fever of Unknown Origin (FUO): A Review
}

\author{
AHMED JU ${ }^{\mathrm{a}}$, RAHIM MA ${ }^{\mathrm{b}}$, HOSSAIN MD $^{\mathrm{c}}$, AHMED AKMS ${ }^{\mathrm{d}}$, MUSA AKM ${ }^{\mathrm{e}}, \mathrm{UDDIN} \mathrm{KN}^{\mathrm{f}}$
}

\begin{abstract}
Fever of unknown origin (FUO) is a diagnostic challenge for clinicians. Disorders presenting as fever of unknown origin are varied and extensive. Clinicians often find themselves hopeless with a patient with FUO and try to catch a straw by doing every conceivable test and run therapeutic trials in order to diagnose all of the myriad causes of FUO that are in fact part of the differential diagnosis of FUO in general. The main difficulty with diagnostic testing in patients with FUO is that it is unfocused. All disorders have a specific pattern of organ involvement. In a patient with FUO, there are almost always one or more clues from the
\end{abstract}

\section{Introduction}

Clinicians commonly refer to a febrile illness without an initially obvious etiology (sometimes called fever without localizing signs) as fever of unknown origin (FUO). This usage is not accurate. Most febrile illnesses either resolve before a diagnosis can be made or develop distinguishing characteristics that lead to a diagnosis. FUO refers to a prolonged febrile illness without an established etiology despite intensive evaluation and diagnostic testing. The

a. Dr. Jamal Uddin Ahmed, FCPS (Medicine). Registrar, Internal Medicine \& Pulmonology, Ibrahim Medical College and BIRDEM General Hospital, Shahbag, Dhaka 1000.

b. Dr. Muhammad Abdur Rahim, FCPS (Medicine). Registrar, Internal Medicine \& Rheumatology, Ibrahim Medical College and BIRDEM General Hospital, Shahbag, Dhaka 1000.

c. Dr. Md. Delwar Hossain, MD (Chest), Associate Professor, Internal Medicine \& Pulmonology, Ibrahim Medical College and BIRDEM General Hospital, Shahbag, Dhaka 1000.

d. Dr. AKM Shaheen Ahmed, FCPS (Medicine), MCPS (Medicine). Associate Professor, Internal Medicine \& Oncology, Ibrahim Medical College and BIRDEM General Hospital, Shahbag, Dhaka 1000.

e. Prof. AKM Musa, FCPS (Medicine), MCPS (Medicine), DTCD. Professor of Medicine \& Pulmonology, Ibrahim Medical College and BIRDEM General Hospital, Shahbag, Dhaka 1000.

f. Prof. Khwaja Nazim Uddin, FCPS (Medicine), FRCP, FACP. Professor of Medicine \& Rheumatology and Head, Internal Medicine, Ibrahim Medical College and BIRDEM General Hospital, Shahbag, Dhaka 1000

Address of correspondence: Dr. Jamal Uddin Ahmed, FCPS (Medicine), Registrar, Internal Medicine \& Pulmonology, Ibrahim Medical College and BIRDEM General Hospital, Shahbag, Dhaka 1000. E-mail: jmldollar@gmail.com

Received: 20 August 2012 Accepted: 10 October 2012 history, physical examination, or nonspecific laboratory tests that suggest a particular diagnosis or at least limit diagnostic possibilities. It is worthy to remember that fever of unknown origin is more often caused by an atypical presentation of a common entity than by a rare disorder. Thus a focused diagnostic approach can minimize the miseries of both the clinician \& the patient

Key words: Fever of unknown origin, focused diagnostic approach

(Birdem Med J 2013; 3(1): 27-34)

definition of FUO, derived by Petersdorf and Beeson in 1961 from a prospective analysis of 100 cases, has long been the clinical standard ${ }^{1}$. It defines FUO as fever higher than $38.3^{\circ} \mathrm{C}$ on several occasions with duration of fever for at least three weeks and uncertain diagnosis after one week of study in the hospital. This definition has been modified over the years to take into account the change in diagnostic modalities and the proportion of patients evaluated for FUO in the ambulatory versus the inpatient setting. Expansion of the definition has also been suggested to include nosocomial, neutropenic, and HIVassociated fevers that may not be prolonged ${ }^{2}$. Thus the new definition by Durack and Street has eliminated the in-hospital evaluation requirements with 3 outpatient visits, or 3 days evaluation in hospital ${ }^{3}$.

The prevalence of FUO in hospitalized patients is reported to be $2.9 \%$. A meta-analysis of 11 studies $^{4}$ of FUO indicate that the spectrum of disease includes "no diagnosis" (19\%), infections (28\%), inflammatory diseases (21\%), and malignancies (17\%). Deep vein thrombosis (3\%) and temporal arteritis in the elderly (16\%-17\%) were important considerations. True FUOs are uncommon. In 1930s 70\% of FUO remained undiagnosed which has become 5-10\% in 2000. Undiagnosed FUO patients generally have good outcome. Eighty percent patients recover spontaneously within 4 weeks.

\section{Classification of FUO}

FUOs fall into four general categories (Table-I). The relative frequency of the causes of FUO in each category is the basis for a phased diagnostic approach. 
Table-I

\begin{tabular}{|c|c|c|}
\hline \multicolumn{3}{|c|}{ Classification of $\mathrm{FUO}^{3}$} \\
\hline Category of FUO & Definition & Common etiologies \\
\hline Classic & $\begin{array}{l}\text { Temperature }>38.3^{\circ} \mathrm{C}\left(100.9^{\circ} \mathrm{F}\right) \\
\text { Duration of }>3 \text { weeks } \\
\text { Evaluation of at least } 3 \text { outpatient visits or } \\
3 \text { days in hospital }\end{array}$ & Infection, malignancy, collagen vascular disease \\
\hline Nosocomial & $\begin{array}{l}\text { Temperature }>38^{\circ} \mathrm{C} \\
\text { Patient hospitalized } \geq 24 \text { hours but no fever } \\
\text { or incubating on admission } \\
\text { Evaluation of at least } 3 \text { days }\end{array}$ & $\begin{array}{l}\text { Clostridium difficile enterocolitis, drug-induced, } \\
\text { pulmonary embolism, septic thrombophlebits, } \\
\text { Sinusitis }\end{array}$ \\
\hline $\begin{array}{l}\text { Immune deficient } \\
\text { (neutropenid }\end{array}$ & $\begin{array}{l}\text { Temperature }>38^{\circ} \mathrm{C} \\
\text { Neutrophil count } \leq 500 \text { per } \mathrm{mm}^{3} \\
\text { Evaluation of at least } 3 \text { days }\end{array}$ & $\begin{array}{l}\text { Opportunistic bacterial infections, } \\
\text { aspergillosis, candidiasis, herpes virus }\end{array}$ \\
\hline HIV-associated & $\begin{array}{l}\text { Temperature }>38^{\circ} \mathrm{C} \\
\text { Duration of }>4 \text { weeks for outpatients, } \\
>3 \text { daysfor inpatients } \\
\text { HIV infection confirmed }\end{array}$ & $\begin{array}{l}\text { Cytomegalovirus, Mycobacterium avum- } \\
\text { intraceflulare complex, Pneumocystis } \\
\text { cadnll pneumonia, drug-induced, } \\
\text { Kaposis sarcoma, lymphoma }\end{array}$ \\
\hline
\end{tabular}

Causes of FUO:

In the classic article on FUO by Petersdorf, infectious diseases were the single largest category responsible for FUOs ${ }^{\mathbf{1}}$. Years later, Petersdorf again reported on the relative incidences of disorders causing FUOs and found that neoplasms had replaced infections as the most common category causing FUOs ${ }^{5}$. Since the 1990 s, there have been further changes in the relative distribution of causes responsible for FUOs ${ }^{6}$. The changes in the relative distribution of entities responsible for FUO are primarily due to changes in diagnostic testing rather than to a major shift in the relative incidence of general categories. The biggest change in diagnostic categories is related to a decrease in the relative proportion of collagen vascular diseases causing FUO. Because of accurate, early diagnostic testing for collagen vascular diseases, those accompanied by fevers of prolonged duration do not remain undiagnosed, and therefore do not fulfill the criteria of an FUO. The collagen vascular diseases that have remained important causes of FUO are those for which no serological tests are available, that is, polymyalgia rheumatica (PMR)/temporal arteritis, late onset rheumatoid arthritis (LORA), and juvenile rheumatoid arthritis (adult Still's disease).

\section{Table-II}

\begin{tabular}{|c|c|}
\hline \multicolumn{2}{|c|}{ Common etiologies of $\mathrm{FUO}^{7}$} \\
\hline INFECTIONS & AUTOIMMUNE CONDITIONS \\
\hline Tuberculosis (especially & Adult Still’s disease \\
\hline extrapulmonary) & Polymyalgia rheumatica \\
\hline Abdominal abscesses & Temporal arteritis \\
\hline Pelvic abscesses & Rheumatoid arthritis \\
\hline Dental abscesses & Rheumatoid fever \\
\hline Endocarditis & Inflammatory bowel disease \\
\hline 0steomyelitis & Reiter's syndrome \\
\hline Sinusitis & Systemic lupus erythematosus \\
\hline Cytomegalovirus & Vascu litides \\
\hline Epstein-Barn virus & Miscellaneous \\
\hline Human immunodeficiency & Drug-induced fever \\
\hline virus & Complications from cirrhosis \\
\hline Lyme disease & Factitious fever \\
\hline Prostatitis & Hepatitis (alcahaiic, \\
\hline Sinusitis & granulamatous, or lupoid) \\
\hline MALIGNANCIES & Deep venous thrombosis \\
\hline Chronic leukemia & Sarcoidosis \\
\hline \multicolumn{2}{|l|}{ Lymphoma } \\
\hline \multicolumn{2}{|l|}{ Metastatic cancers } \\
\hline \multicolumn{2}{|l|}{ Renal cell carcinoma } \\
\hline \multicolumn{2}{|l|}{ Colon carcinoma } \\
\hline \multicolumn{2}{|l|}{ Hepatoma } \\
\hline \multicolumn{2}{|l|}{ Myelodysplastic syndromes } \\
\hline \multicolumn{2}{|l|}{ Pancreatic carcinoma } \\
\hline Sarcomas & \\
\hline
\end{tabular}




\section{Evaluation of the Patient with FUO}

The main diagnostic difficulty with FUO is a comprehensive, efficient and effective diagnostic approach. Unfortunately, often this has only resulted in excessive diagnostic testing to rule out every disorder causing FUO. An unfocused approach has the effect of incurring unnecessary expense, inconveniencing patients, and delaying or obscuring the FUO diagnostic work-up. The undesirable effect of the "shotgun approach" to diagnostic testing is that it under uses the FUO tests appropriate for the most likely diagnostic categories, and it over tests for unlikely diagnoses ${ }^{8}$.

The diagnostic approach to FUOs may be considered as consisting of three phases ${ }^{9,10}$. The initial phase consists of the initial FUO history and physical examination including confirming the presence of fever \& documenting it's pattern, then doing some nonspecific laboratory tests (Table III). This phase provides the clinician with a general sense of whether the FUO is likely to be caused by an infection or by a rheumatic, inflammatory or neoplastic disorder. After the history, physical exam, and nonspecific laboratory tests, further tests should be based on localizing the disease process anatomically to determine its organ system distribution, which in turn is critical in defining differential diagnostic possibilities.

The second phase involves re-evaluating the patient using a focused FUO history and physical examination and additional nonspecific and specific laboratory tests 12. The focused FUO evaluation has the effect of narrowing diagnostic possibilities and eliminating possibilities from further diagnostic consideration (Table IV, V, VI). Among different tests done for FUO, perhaps the most underutilized and undervalued test is serum ferritin levels. Elevations of serum ferritin levels are often ignored or explained away as being due to ferritin acting as an "acute phase reactant." In a patient with FUO, by definition, the process is no longer acute, and elevations in the serum ferritin level take on a very different significance ${ }^{13}$. Elevated serum ferritin levels may suggest certain collagen vascular diseases, for example, systemic lupus erythematosus (SLE), juvenile rheumatoid arthritis (JRA), or temporal arteritis ${ }^{14,15}$. Ferritin levels may also be elevated in a variety of myeloproliferative disorders as well as with any malignancy. Importantly, elevated ferritin levels in the FUO context strongly argue against an infectious etiology.

During evaluating a patient with FUO, clinicians have to rule out the big and little 3 causes of FUO. The big 3 are - infection, malignancy and collagen diseases and the little 3 are - drug fever, factitious fever and habitual hyperthermia. Drug fever is an important but often forgotten cause of $\mathrm{FUO}^{16}$. Clues to drug fever include progressive eosinophilia and subsidence of fever within 48 hours of withdrawal of suspected drug.

Table-III

Nonspecific tests as a guide for further testing in $\mathrm{FUO}^{11}$

For all FUO categories

$\mathrm{CBC}$

ESR

LFTs

Chest $\mathrm{X}$ ray

ANA

UA

Routine blood cultures

$\mathrm{CBC}$

Leukocytosis $\rightarrow$ neoplastic and infectious panels

Leukopenia $\rightarrow$ neoplastic infections, and RD panels

Anemia $\rightarrow$ neoplastic, infections, and RD panels

Myelocytes/metamyelocytes $\rightarrow$ neoplastic panel

Lymphocytosis $\rightarrow$ neoplastic and infectious panels Lymphopenia

Atypical lymphocytes

Eosinophilia $\rightarrow$ neoplastic, RD, and infectious panels

Basophilia $\rightarrow$ neoplastic panel

Thrombocytosis $\rightarrow$ neoplastic, infectious, and RD panels

Thrombocylopenia $\rightarrow$ neoplastic, infectious, \& RD panels

ESR

Highly elevated $\rightarrow$ neoplastic, infectious, and RD panels

LFTs

$\uparrow \mathrm{SGOT/SGPT} \rightarrow \mathrm{RD}$ panel

$\uparrow$ all phosphatases $\rightarrow$ neoplastic and RD panels

ANA

Increased ANA $\rightarrow$ RD panel

Increased $\mathrm{RF} \rightarrow$ infectious and RD panels

Chest $\mathrm{X}$ ray

Any lung parenchymal abnormality/adenopathy/ pleural effusion $\rightarrow$ neoplastic, infectious, or RD panels

Blood cultures

Imaging studies

Abbreviations: ANA, antinuclear antibodies; CBC, complete blood count; ESR, erythrucyte sedimentation rate; LFTs, liver function tests; RD, rheumatic disease; SCOT/SGPT, serum glutamicoxaktacetic transaminase/serum glutamic pyruvate transaminase; UA, urine analysis. 


\section{TableIV}

\section{Diagnostic clues to infectious disease as cause of $\mathrm{FUO}^{23,24}$}

History

Fatigue (any chronic infection)

Weight loss (abscesses, HIV, TB, SBE)

Night sweats (abscesses, HIV, TB, SBE)

Headache (typhoid fever, TB, brucellosis, HIV)

Mental confusion (brucellosis, TB, chronic viral/parasitic CNS infections, HIV, CSF)

Sudden vision loss (SBE, brain abscess)

CVA (TB, SBE)

Tongue pain (relapsing fever)

Shoulder pain (subdiaphragmatic abscess)

Arthralgias (LGV, Whipple's disease, rat bite fever, brucellosis, HIV)

Cough (TA, TB)

Heart murmur (SBE)

Back pain (TB, brucellosis, SBE)

Thigh pain (brucellosis)

Early satiety (brucellosis, splenic abscess, typhoid fever)

Animal contact (brucellosis, typhoid fever, O fever, CSF, psittacosis, rat bite fever)

IVDA/blood transfusions (CMV, HIV)

Physical findings

Relative bradycardia (typhoid fever, leptospirosis, psittacosis, brucellosis, malaria)

Epistaxis (psittacosis, relapsing fever)

Conjunctivitis (TB, CSF)

Conjunctival suffusion (relapsing fever)

Subconjunctival hemorrhage (SBE) Uveitis (TB)

Adenopathy

Localized (toxoplasma, CSF, HIV)

Generalized (HIV, EBV, CMV, TB, LGV, brucellosis)

Heart murmur (SBE)

Trapezius tenderness (subdiaphragmatic abscess)

Spinal tenderness (SBE, brucellosis, typhoid fever)

Hepatomegaly (relapsing fever, typhoid fever, Q fever)

Splenomegaly (TB, SBE, brucellosis, EBV, CMV, psittacosis, relapsing fever, typhoid fever)

Thigh tendemess (brucellosis)

Thrombophlebitis (psittacosis)

Epididymoonchitis (TB, brucellosis, leptospirosis, EBV)

Arthritis (rat bite fever, brucellosis, osteomyelitis, typhoid fever, Whipple's disease)

Nonspecific laboratory findings

CBC

Leukopenia (HIV, TB, brucellosis, typhoid fever)

Lymphopenia (HIV, TB)

Lymphocytosis (TB, EBV, CMV, toxoplasmosis)

Monocytosis (SBE, TB, brucellosis, CMV)

Atypical large/bizarre lymphocytes (toxoplasmosis, CMV, EBV)

Thrombocytopenia (HIV, CMV, RSV, relapsing fever)

ESR

Thrombocytosis (abscess, osteomyelitis, SBE, TB)

Highly elevated ESR $>100$ mm/hr (abscess, osteomyelitis, SBE)

Rheumatoid factor SPEP

Increased rheumatoid factors (SBE)

Polyclonal gammopathy (HIV)

Increased SGOT/SGPT (EBV,CMV, O fever, psittacosis, to)*plasmosis, relapsing fever, brucellosis)

Increased alkaline phosphatase (TB)

Abbreviations: CBC, complete blood count; CMV, cytomegalovirus; CNS, central nervous system; CSF, cat scratchfever; CVA, carriiavascularaccident; EBV, Epstein-Ban' virus; ESR, erythrocyte sedimentation rate; HCV, hepatitis C virus; HIV, human immunodeficiency virus; LGV, lympho-granuloma venereum; RSV, respiratory synaytial Virus; SBE, subacute bacterial endocarditis; SOOT/SGPT, serum glutamiccxaloaoetictransaminase/ serum glutamic pyruvate transaminase; SPEP, serum protein electrophoresis; TA, temporal arteritis; TB, tuberculosis. 


\section{Table-V}

\section{Diagnostic clues to malignant disorders as cause of FUO ${ }^{23,24}$}

History

Fatigue (any neoplastic disorder)

Decreased appetite/weight loss (any neoplastic disorder)

Headache (primary/metastatic CNS neoplasms)

Cough (pulmonary neoplasms)

Night sweats (any neoplastic disorder)

Physical findings

Relative bradycardia (lymphomas)

Stemal tendemess (preleukemias, myeloproliferative disorders, lymphoreticular malignancies)

Pleural effusion (lymphomas, pulmonary neoplasms, metastases)

Heart murmur (atrial myoma)

Hepatomegaly (hematoma, metastases, lymphomas)

Splenomegaly (leukemias, lymphomas)

Ascites (peritoneal/omental metastases)

Lymphadenopathy (lymphomas, CLL)

Epididymoorchitis (lymphoma)

Nonspecific laboratory tests

$\mathrm{CBC}$

Leukocytosis (MPD, CLL)

Leukopenia (lymphoreticular malignancies)

Anemia (any malignancy)

Myocytes/melamyelocytes/nucleated RBCs/ “teardrop RBCs” (neoplastic bone marrow involvement)

Atypical (small/uniform) lymphocytes (CLL)

Eosinophilia (MPD, leukemias, lymphomas)

Basophilia (MPD, leukemias, lymphomas)

Thrombocytopenia (any malignancy with bone marrow involvement)

Thrombocytosis (any malignancy)

ESR

Highly elevated ESR > 100 mm/hr (any neoplastic disorder)

LFTs

Increased alkaline phosphatase (hepatomas, lymphomas, liver metastases)

SPEP

Increased monoclonal gammopathy (multiple myeloma)

Increased a1/a 2 globulins (lymphomas)

Serum ferritin

Increased ferritin levels (MPD, any malignancy)

Abbreviations: GBC, complete blood count; CLL, chronic lymphocytic lymphoma; CNS, central nervous system; E SR, erythrocyte sedimentation rate; LFT, liver function tests; MPD, myeloprolderative disorders; RBG, red blood cells; SPEP, serum protein electrophoresis. 


\section{Table-VI}

\section{Diagnostic clues to rheumatic disorders as cause of FUO 23,24}

History

Dry eyes (LORA, SLE)

Watery eyes (PAN)

Vision disorders/eye pain (Takayasu's arteritis, TA)

Headache (temporal pain, TA)

Neck pain (jaw pain, adult JRA) Dry cough (TA)

Abdominal pain (PAN, SLE)

Myalgias/arthralgias (PAN, adult JRA, FMF, LORA, SLE) generalized localized

Physical findings

Eyes

Band keratopathy (adult JRA)

Conjunctivitis (SLE)

Uveitis (adult JRA, sarcoidosis, SLE)

Dry eyes (LORA, SLE)

Watery eyes (PAN)

Fundi [cytoid bodies” (SLE), “candlewax drippings” (sarcoidosis)]

Lymphadenopathy (Kikuchi’s disease, adult JRA)

Splenomegaly (SLE, LORA, sarcoidosis, Kikuchi’s disease)

Epididymoorchitis (PAN)

Nonspecific laboratory tests

Blood tests (all rheumatic disorders)

$\mathrm{CBC}$

Leukopenia (SLE)

Lymphopenia (sarcoidosis/lymphoma syndrome)

Eosinophilia (sarcoidosis, PAN) Thrombocytopenia (SLE)

ESR

Highly elevated ESR $>100 \mathrm{~mm} / \mathrm{hr}$ (all rheumatic disorders) LFTs

Increased SGOT/SGPT (Kikuchi's disease, adult JRA)

Increased alkaline phosphatase (PAN)

SPEP

Polyclonal gammopathy (SLE)

Increased a1,a2 globulins (SLE)

Ferritin

Increased ferritin levels (adult JRA, SLE, TA)

Abbreviations: ESR, erythrocyte sedimentation rate; FMF, familial Mediterranean fever; JRA, juvenile rheumatoid arthritis; LFTs, liver function tests; LORA, late onset rheumatoid arthritis; PAN, periarterifis nodosa; SGOT/SGPT, serum glutamicoxaloacetic transaminase/serum glutamic pyruvate transaminase; SLE, systemic lupus erythematosus; SPEP, serum protein electrophoresis; TA, temporal arthritis. 
Here is a proposed algorithm to approach to a patient with $\mathrm{FUO}^{25}$.

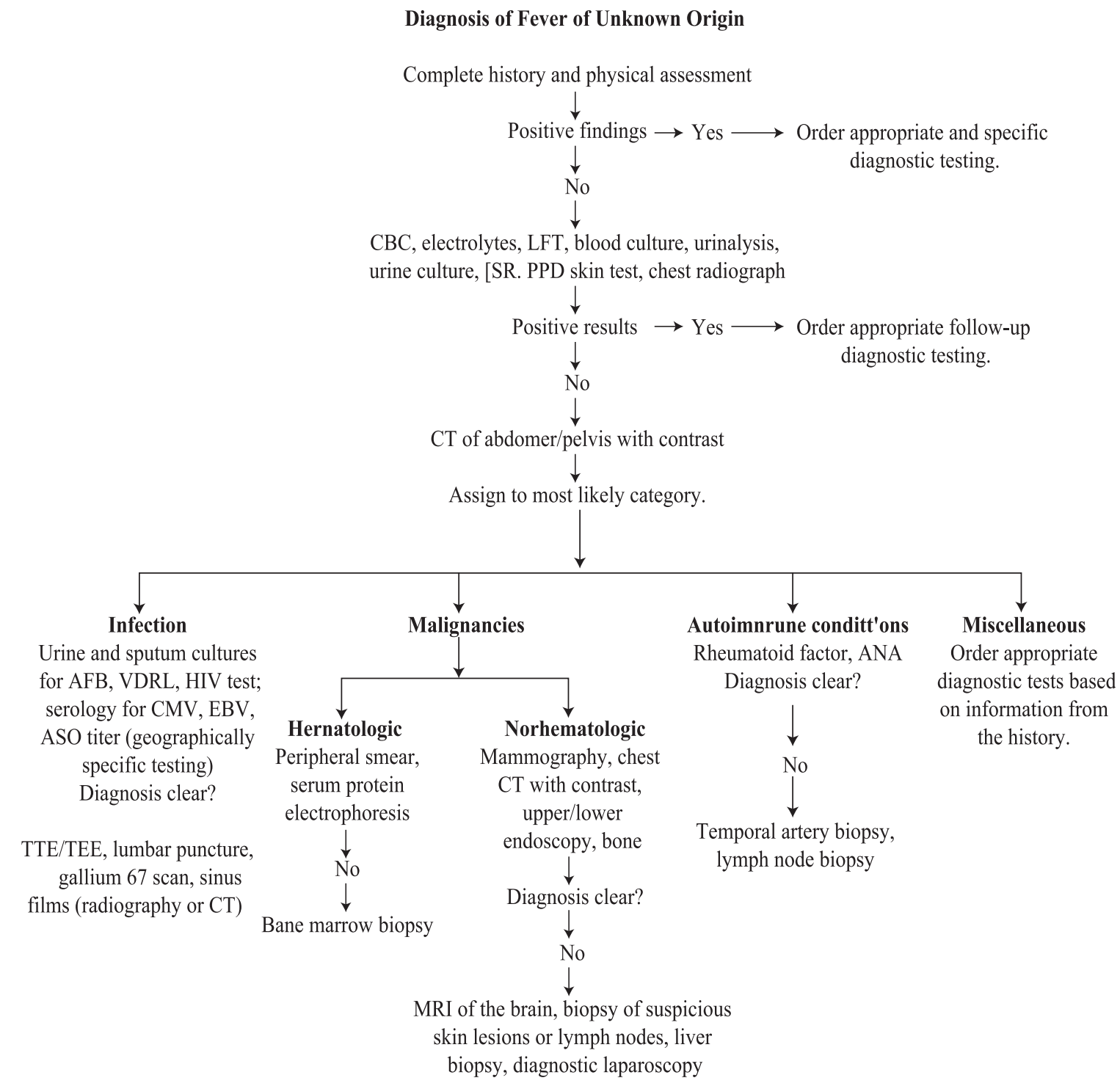

Abbreviations: $(\mathrm{CBC}=$ complete blood count; LFT = liver function test; $\mathrm{ESR}=$ erythrocyte sedimentation rate; $\mathrm{PPD}=$ purified protein derivative; $\mathrm{CT}=$ computed tomography; $\mathrm{AFB}=$ acid-fast bacilli; $\mathrm{HIV}=$ human immunodeficiency virus; $\mathrm{CMV}=$ cytomegalovirus; $\mathrm{EBV}=$ Epstein-Barr virus; $\mathrm{ASO}$ = antistreptolysin-O antibodies; $\mathrm{ANA}=$ antinuclear antibody; TTE = transthoracic echocardiography; TEE = transesophageal echocardiography; MRI = magnetic resonance imaging)

In the third phase the approach has to be more specific and invasive. More specific tests including invasive tests like biopsy or laparoscopy, advanced imaging is required ${ }^{17,18}$. In this state the question of therapeutic trial comes in which is a controversial issue ${ }^{19}$. Empiric therapy plays a limited role in $\mathrm{FUO}^{20}$. Therapeutic trials of antimicrobials or glucocorticoids, while tempting in the effort to "do something," rarely establish a diagnosis. In addition, the diagnostic yield of blood cultures and cultures of biopsy material will be reduced following the initiation of antibiotics ${ }^{21}$. Antimicrobial agents could be expected to suppress, but not cure, an infectious process such as an occult abscess since adjunctive drainage would usually be required. Cunha 
in 1996 recommended $^{2}$ empiric therapy for only 4 situations: antibiotics for culture-negative endocarditis, low-dose corticosteroids for presumed temporal arteritis, antituberculous drugs for suspected military tuberculosis in elderly patients, and naproxen (Naprosyn) for suspected neoplastic fever ${ }^{22}$.

\section{Conclusion}

FUOs usually are limited by their progression and are self-terminating or are terminated with effective therapy. Some causes of FUO are prone to recurrence. Using a focused diagnostic approach a three-tiered system leaves very few disorders undiagnosed. Most of the common causes of FUO are diagnosed during the initial FUO evaluation. The focused FUO evaluation should be able to diagnose less common and obscure disorders associated with prolonged and perplexing fevers. It is worthy to remember the saying - "Some fevers remain of unknown origin and represent a source for humility on the part of the diagnostician, but may at the same time serve as an impetus for continued research."

\section{References}

1. Petersdorf RG, Beeson PB. Fever of unexplained origin: report on 100 cases. Medicine 1961; 40: 1-30.

2. Cunha BA. Fever of unknown origin. Infect Dis Clin North Am 1996; 10: 111-27.

3. Durack DT, Street AC. Fever of unknown originreexamined and redefined. Curr Clin Top Infect Dis 1991; 11: 35-51.

4. Mourad O, Palda V, Detsky AS. A comprehensive evidencebased approach to fever of unknown origin. Arch Intern Med. 2003; 163 (5): 545-51.

5. Petersdorf RF. Fever of unknown origin: an old friend revisited. Arch Intern Med 1992; 152: 21-25

6. Knockaert DC, Vanneste LJ, Vannester SB, et al. Fever of unknown origin in the 1980s: an update of the diagnostic spectrum. Arch Intern Med 1992; 152: 51-55

7. De Kleijn EM, Vandenbroucke JP, van der Meer JW. Fever of unknown origin (FUO). A prospective multicenter study of 167 patients with FUO, using fixed epidemiologic entry criteria. The Netherlands FUO Study Group. Medicine 1997;76: 392- 400.

8. Tumulty PA. Topics in clinical medicine: The patient with fever of undetermined origin. Johns Hopkins Med J 1967; 120: 95-106.

9. Esposito AL. Planning and proceeding with the diagnostic evaluation. In: Murray HW, editor. Fever of undetermined origin. Mount Kisco (NY): Future Publishing; 1983: 14155.
10. Cunha BA. Fever of unknown origin: a focused diagnostic approach. In: Cunha BA, editor. Fever of unknown origin. New York: Informa Healthcare; 2007: 9- 16.

11. Cunha BA. Diagnostic significance of nonspecific laboratory abnormalities in infectious diseases. In: Gorbach SL, Bartlett JG, Blacklow NE, eds. Infectious Diseases. 3rd ed. Philadelphia: Lippincott Williams and Wilkins, 2004:158-65.

12. Tumulty PA, editor. The patient with fever of unknown origin (FUO): the effective clinician. Philadelphia: WB Saunders; 1973: 137-70.

13. Krol V, Cunha BA. Diagnostic significance of serum ferritin levels in infectious and noninfectious diseases. Infect Dis Pract 2003; 27:196-97.

14. Beyan E, Beyan C, Demirezer A, et al. The relationship between ferritin levels and disease activity in systemic lupus erythematosus. Scand J Rheumatol 2003; 32: 225-28.

15. Cunha BA, Parchuri S, Mohan S. Fever of unknown origin: temporal arteritis Presenting with persistent cough and elevated serum ferritin levels. Heart Lung 2006; 35: 112-16.

16. Johnson DH, Cunha BA. Drug Fever. Infect Dis Clin North Am. 1996; 10: 85-91

17. Pacios E, Alcala L, Ruis-Serrano MJ, et al. Evaluation of bone marrow and blood Cultures for the recovery of mycobacteria in the diagnosis of disseminated Mycobacterial infections. Clin Microbiol Infect 2004; 10(8):734-37.

18. Meller J, Ivancevic V, Conrad M, et al. Clinical value of immunoscintigraphy in Patients with fever of unknown origin. J Nucl Med 1998; 39(7):1248-53.

19. Hurley DL. Fever in adults: what to do when the cause is not obvious. Postgrad Med1983; 74(5):232-44

20. Plaisance KI, Mackowaik PA. Antipyretic therapy: physiologic rationale, Diagnostic implications, and clinical consequences. Arch Intern Med 2000; 160(4):449-56.

21. Ah Kine D, Tijani SO, Parums DV, et al. Effects of prior steroid treatment on Temporal artery biopsy findings in giant cell arteritis. Br J Ophthalmol 2002; 86(5): 530-32.

22. Chang JC, Gross HM. Utility of naproxen in the differential diagnosis of fever of Undetermined origin in patients with cancer. Am J Med 1984; 76(4):597-603.

23. Sen P, Louria DB. Noninvasive and diagnostic procedures and laboratory methods. In: MurrayHW, ed. FUO: Fever of Undetermined Origin. Mount Kisco, NY, Futura Publishing, 1983:159-90.

24. Ravel R. Clinical Laboratory Medicine. 6th ed. New York: Mosby, 1995

25. Bleeker-Rovers CP, Vos FJ, de Kleijn EM, et al. A prospective multicenter study on fever of unknown origin: the yield of a structured diagnostic protocol. Medicine 2007; 86: 26-38. 